\title{
Lesiones de astrágalo que pueden ser confundidas con esguinces de tobillo
}

\author{
Talus lesions that can be mistaken for ankle sprains
}

\author{
José Luis Muñoz Sánchez, Javier Navarro Marruedo \\ Grado en Podología. Universidad Complutense de Madrid. \\ joseguis16@hotmail.com \\ javinavarro46@hotmail.com
}

Correspondencia:
Jose Luis Muñoz Sánchez
C/ Iglesia 14
E-23360 La Puerta De Segura (Jaén)
Correo electrónico: joseguis16@hotmail.com; javinavarro46@hotmail.com

Fecha de recepción: 15 de agosto de 2014

Fecha de aceptación: 30 de diciembre de 2014

Los autores declaran no tener ningún tipo de interés económico o comercial.

\section{RESUMEN}

Existen lesiones en el tobillo que pueden dar una clínica y sintomátología similar al esguince de tobillo no siendo uno de estos. Estas lesiones que se asemejan a esguinces de tobillo, suelen darse en pacientes jóvenes y son debidas a lesiones producidas en el astrágalo. Entre las lesiones más frecuentes producidas en el astrágalo, están los síndromes de pinzamiento o compresión, las fracturas y la osteocondritis disecante. La osteocondritis disecante de astrágalo fue descrita por primera vez en 1888 por Konig como cuerpos libres en la articulación de la rodilla por la necrosis espontánea del hueso, desde entonces, ha sido estudiada y clasificada por diversos autores como Berdnt y Harty en 1959. Para estas lesiones existen tratamientos conservadores así como diversas técnicas quirúrgicas en caso de ser necesarias. Entre las técnicas quirúrgicas más empleadas para el tratamiento de la osteocondritis disecante de astrágalo están la artrotomía y la artroscopia.

Palabras clave: astrágalo; pie; esguinces; tobillo; osteocondritis.

\section{ABSTRACT}

There are ankle injuries that can give similar symptoms and clinical sprained ankle,to not being one of these. These lesions resemble ankle sprains, usually occur in young patients and are due to lesions in the talus. Among the most common injuries in the talus syndromes are pinching or compression, fractures and osteochondritis dissecans. Osteochondritis dissecans of the talus was first described in 1888 by Konig as loose bodies in the knee joint by spontaneous bone necrosis, since then, has been studied and classified by various authors as Berdnt and Harty in 1959. For these lesions are different conservative treatments and surgical techniques if needed. Among the most widely used for the treatment of osteochondritis dissecans of the talus surgical techniques are arthrotomy and arthroscopy. Keywords: talus; foot; sprains; ankle; osteochondritis.

Sumario: 1. Introducción. 2. Osteocondritis disecante de astrágalo. 3. Diagnóstico. 4. Tratamiento. 5. Técnica de la artrotomía de tobillo. 6. Técnica de Thompson y Loomer. 7. comclusiones Bibliografía.

Referencia normalizada: Muñoz Sánchez, J.L., Navarro Marruedo, J. Lesiones de astrágalo que pueden ser confundidas con esguinces de tobillo. Rev. Int. Cienc. Podol. 2015; 9(2): 106-116. 


\section{INTRODUCCIÓN}

Los esguinces de tobillo están entre las lesiones más comunes en el deporte, estas ocurren hasta en el $70 \%$ de los jugadores de baloncesto adolescentes de educación secundaria ${ }^{1}$. Aunque los esguinces de tobillo sean de menor importancia, hasta un tercio de los atletas con estas lesiones pueden perder dos o más semanas de competición ${ }^{2}$.

Son muchas las lesiones de la articulación del tobillo que se parecen al esguince de tobillo, incluyendo neoplasias como el osteoma osteoide, granuloma eosinófilico y la sinovitis pigmentada vellonodular (Figura 1). Snyder et al. ${ }^{3}$ y $\mathrm{O}^{\prime} \mathrm{Neil}$ y Micheli ${ }^{4}$ informaron de que existia una alta incidencia de coaliciónes tarsales (Figura 2) asociadas con esguinces de tobillo en niños y adolescentes, incluyendo las coaliciones fibrosas, coaliciones cartilaginosas y coaliciones óseas. La fractura osteocondral también puede parecerse al esguince de tobillo. Cuando existen síntomas de esguince de tobillo que no se resuelven al cabo de las 4 o 5 semanas de tratamiento conservador, es recomendable valorar una tomografía computarizada y una evaluación adicional para descartar coalición tarsal o fractura osteocondral del astrágalo.

Los síndromes de pinzamieno o compresión, descritos como "crestas osteocondrales"

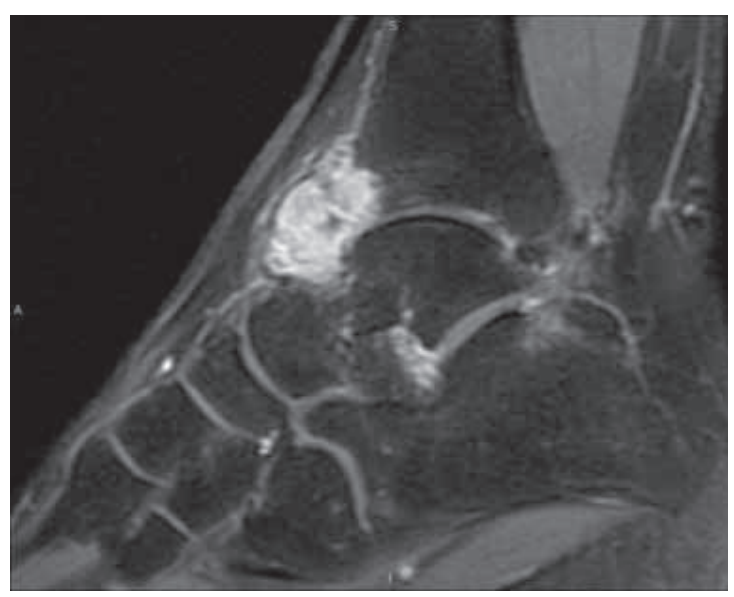

Figura 1. La sinovitis pigmentada vellonodular erosiona la porción superior del cuello del astrágalo causado síntomas de esguince de tobillo en atletas jóvenes. o "exostosis" por O'Donoghue ${ }^{5}$ ocurren en atletas adolescentes. El síndrome de pinzamiento anterior se produce con frecuencia en corredores y saltadores dándose comúnmente en la zona distal de la tibia, donde se localiza generalmente en la región anterior y lateral ${ }^{6,7,8}$. El dolor por lo general es el primer síntoma; comienza como una leve molestia volviéndose más agudo y más localizado en la parte frontal del tobillo pudiendo estar limitado el rango de movilidad articular de la articulación del tobillo. Las exostosis sintomáticas suelen ser blandas y pueden palparse cuando tienen un gran tamaño. Las exostosis pueden verse en las radiografías laterales de rutina; En visiones laterales en flexión dorsal y/o flexión plantar también puede verse el contacto tibiotalar; y el ensanchamiento de espacio posterior de la articulación puede ser visible en visiones laterales en carga.

La restricción de actividad física es por lo general suficiente como tratamiento en los pacientes más jóvenes, aunque los pacientes que están en etapas más cercanas e incluso en la adolescencia pueden combinar también un medicamento anti-inflamatorio o una inyección de cortisona. Rara vez es necesaria la artroscopia o artrotomía para la escisión de un espolón óseo o de osteofitos distales de la tibia o cuello del astrágalo (Figura 3).

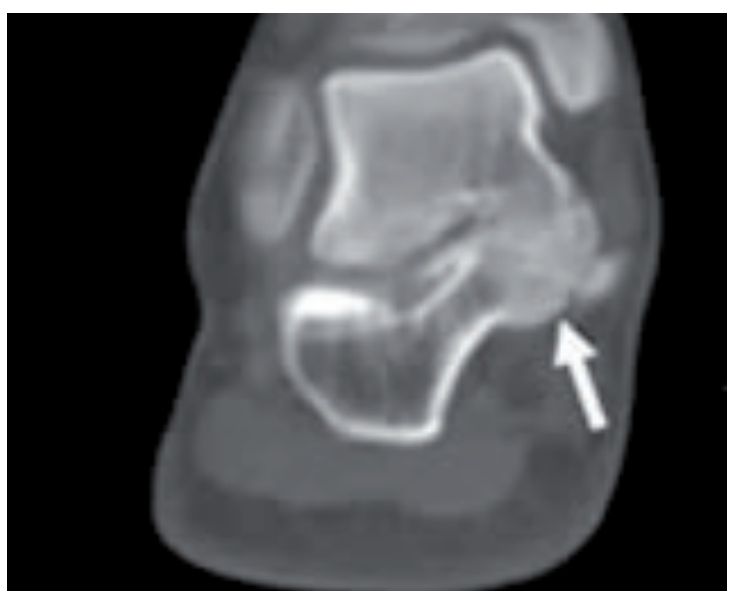

Figura 2. La tomografía computerizada muestra una coalición tarsal entre astrágalo y calcáneo. 


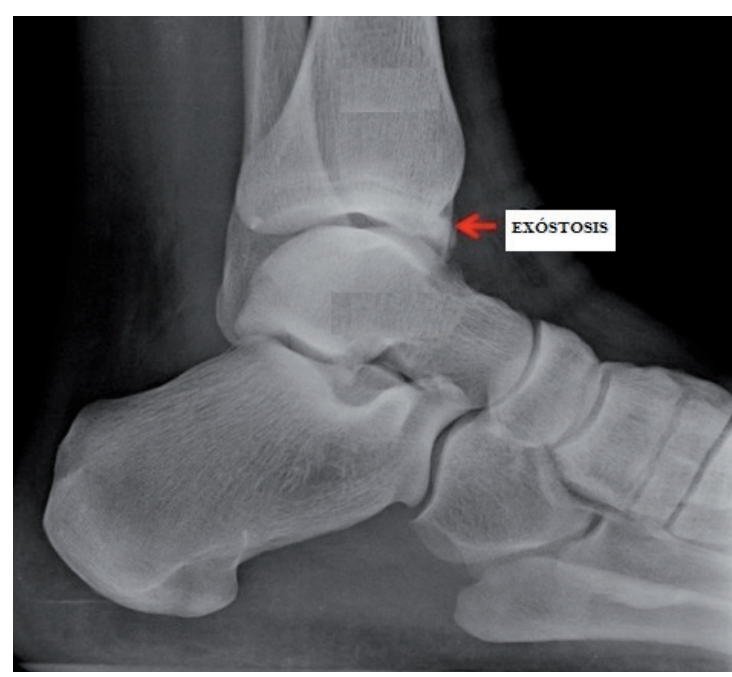

Figura 3. La radiografía lateral muestra una exóstosis distal y lateral tibial.

El síndrome de pinzamiento posterior (síndrome de compresión talar o síndrome del os trigonum) se produce muy a menudo en los bailarines de ballet y corredores ${ }^{9}$. El dolor y sensibilidad se localizan en la cara posterolateral del tobillo detrás de los tendones peroneos. En este síndrome también pueden ser eficaces tratamientos como la restricción de la actividad física y los fármacos antinflamatorios. Brodsky and Khalil $^{9}$ informaron también de la extirpación del hueso os trígono para el síndrome de pinzamiento posterior sintomático en seis bailarines profesionales.

Waller ${ }^{10}$ describió un síndrome de compresión en la esquina anterolateral en la que el dolor se localiza en la región anteroinferior del peroné y la superficie anterolateral del astrágalo, dando el paciente una historia de lesiones por inversión del pie. Este síndrome suele presentar un morfotipo de talón en valgo, y el pie en pronación. El dolor suele mejorar con una posición del pie en supinación teniendo en cuenta que esto puede hacer más suceptible la producción de lesiones por inversión. La mayoría de los autores creen que esta condición es una condromalacia de la cara lateral del astrágalo con una reacción sinovial asociada.

La fractura de la apófisis anterior del calcáneo también puede confundirse con un es-

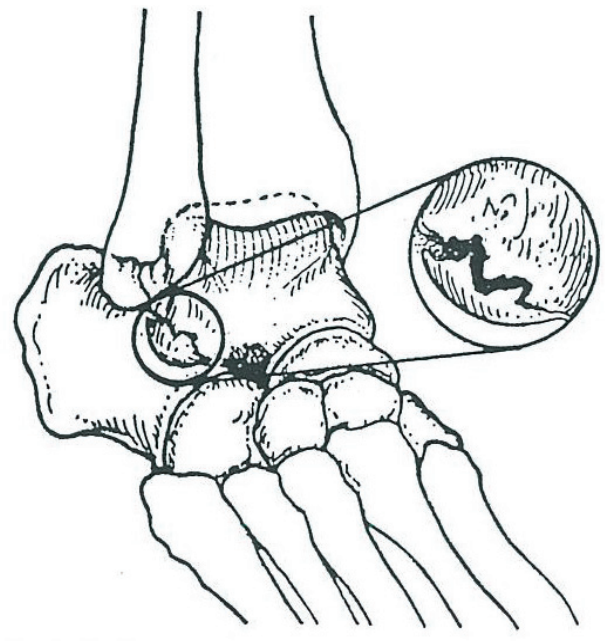

Figura 4. Fractura de la parte inferior del cuerpo lateral del astrágalo.

guince de tobillo. Estas fracturas son de dos tipos: avulsión o compresión. Las fracturas por avulsión suelen ocurrir cuando se produce una lesión de tobillo mediante inversión del mismo, y las fracturas de compresión suelen ocurrir por la abducción forzada del antepie y la consecutiva compresión de la articulación calcáneo-cuboidea. El dolor y la sensibilidad se encuentran en la región del seno del tarso, mas concretamente a $2 \mathrm{~cm}$ por delante y $1 \mathrm{~cm}$ por debajo del ligamento peroneoastragalino anterior, lo que ayuda a distinguir esta lesión de un esguince lateral de tobillo. La fractura por avulsión es la más común y generalmente se trata con inmovilización de la pierna durante 4 semanas mientras que la fractura por compresión es más rara, y a veces los fragmentos articulares desplazados pueden necesitar una reducción abierta y fijación interna.

Las fracturas de estrés o por avulsión ocultas también se producen en esta zona. El hueso os trígono es considerado por muchos autores como una fractura antigua en lugar de un huesecillo separado del hueso, siendo las fracturas del tubérculo posterior adyacente muy difícil de reconocer ${ }^{11,12,13,14}$. Rosemuller ${ }^{15}$ describió el hueso os trígonum como un hueso accesorio del astrágalo, pero Sheperd ${ }^{16}$ creía que era una avulsión del tubérculo posterolateral del as- 
trágalo (fractura de Sheperd). Turner ${ }^{17}$ y Stie$\mathrm{da}^{14}$ entre otros, creian que este osículo surgía de un centro secundario de osificación en la parte posterior del astrágalo que no se unia a él. Watson y Dobas ${ }^{18}$ clasificaron el tubérculo posterior del astrágalo en cuatro etapas: etapa I, proceso talar normal sin significado clínico; etapa II, tubérculo posterolateral del astrágalo aumentado (comúnmente conocido como proceso de Stieda), que puede ser fracturado durante la flexión plantar extrema en lesiones del tobillo; etapa III, hueso accesorio os trígonum que puede ser irritado por microtraumatismos repetitivos y etapa IV, hueso os trígonum con una unión cartilaginosa o sincondrosis con el astrágalo que puede fracturarse con traumatismo agudo. Grogan et al. ${ }^{19}$ usaron especímenes de cadáver, confirmando la exactitud de esta clasificación.

El dolor a la palpación localizado en la región del tubérculo posterolateral del astrágalo es el síntoma mas común de la fractura del tubérculo posterolateral del astrágalo. El edema se localiza a cada lado del tendón de Aquiles. Los pacientes usualmente relatan una historia de flexion plantar en una lesión de tobillo con dolor en la deambulación que se agrava con la flexión plantar del pie. La mejor forma de diferenciar esta

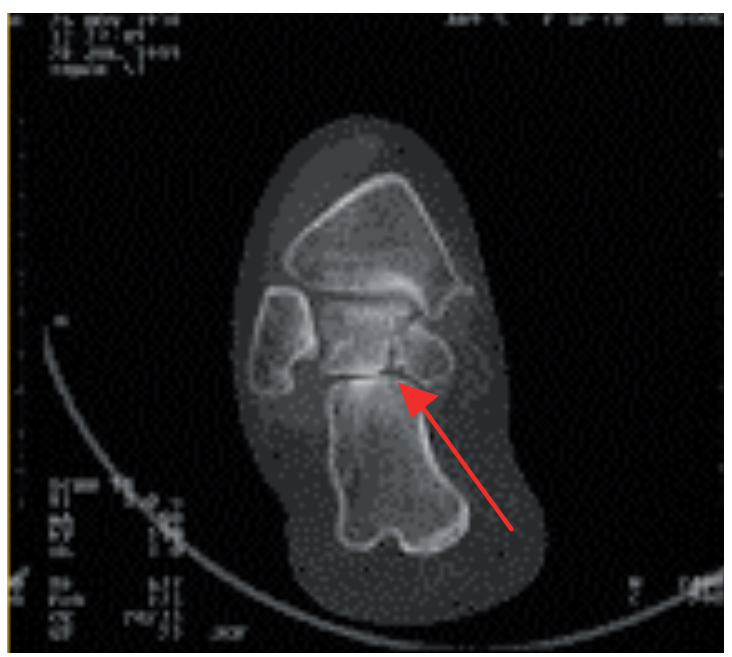

Figura 5. La tomografía computerizada muestra una fractura oculta en el borde inferior del astrágalo. fractura con un centro de osificación secundario no unido es la radiografía lateral.

Otra fractura difícil de evaluar en las radiografías simples, es la de la parte inferior del cuerpo lateral del astrágalo (Figura 4). La gammagrafía ósea es útil para identificar estas fracturas, y en caso de ser positiva debe ser seguida por una tomografía computerizada para determinar la extensión de la fractura (Figura 5). Estas fracturas ocultas en el astrágalo y el tobillo deben ser tratadas, al ser posible sin cirugía porque la exposición quirúrgica es difícil y la morbilidad postoperatoria significativa.

Vuelta a la actividad deportiva. Como con cualquier fractura por avulsión, la curación debe ser casi completa a las 4 a 6 semanas, pudiendo iniciarse el retorno gradual a la actividad a partir de ese momento. Si los síntomas siguen prolongándose deben hacer sospechar de una falta de unión.

\section{OSTEOCONDRITIS DISECANTE DE ASTRÁGALO}

En 1888, Konig ${ }^{20}$ utilizó por primera vez el término: osteocondritis disecante para describir cuerpos libres en la articulación de la rodilla,
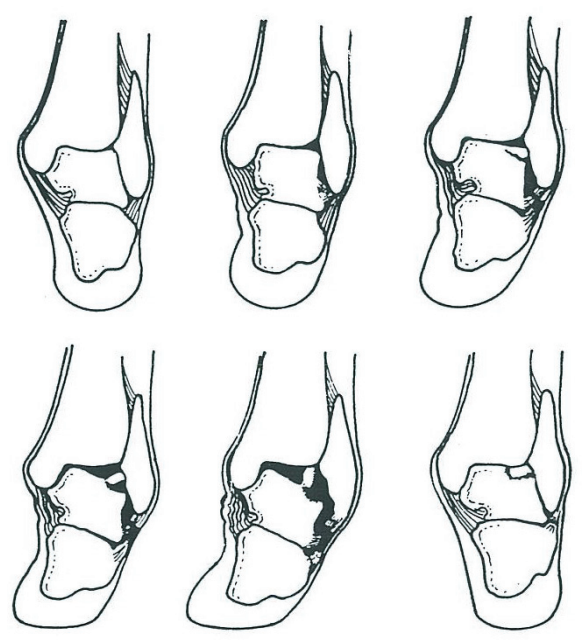

Figura 6. Cuatro estados de la osteocondritis disecante de astrágalo. A, astrágalo normal. B, estado I. C, Estado II. D, Estado III. E, Estado IIIa. F, Estado IV. 
con la teoría de que eran causados por la necrosis espontanea del hueso. En 1922, Kappis ${ }^{21}$ tomó nota de la similitud de las lesiones encontradas en la rodilla y refirió la osteocondritis disecante del tobillo. En 1932, Rendu ${ }^{22}$ informó una fractura intraarticular del astrágalo que parecía ser de naturaleza similar a la lesión de la osteocondritis disecante. En 1953, Roden et al. ${ }^{23}$ reportaron 55 casos de osteocondritis disecante de astrágalo. Estos autores llegaron a la conclusión de que casi todas las lesiones que se producen lateralmente en el astrágalo eran secundarias a un traumatismo rara vez curado espontáneamente y causando más síntomas que las lesiones que se producen en otras localizaciones del tobillo, pudiendo desarrollar una artritis temprana que requeririá operación rápidamente. Por el contrario, un gran porcentaje de las lesiones mediales en su muestra que no eran secundarias a un traumatismo, tuvieron menos síntomas, con frecuencia curaron de forma espontánea, y desarrollaron poco o nada de artrosis, por lo que solo era necesaria la escisión si un cuerpo suelto estaba causando síntomas.

En 1959 Berdnt y Harty ${ }^{24}$ demostraron que tanto las lesiones mediales y laterales de la osteocondritis disecante del astrágalo eran en

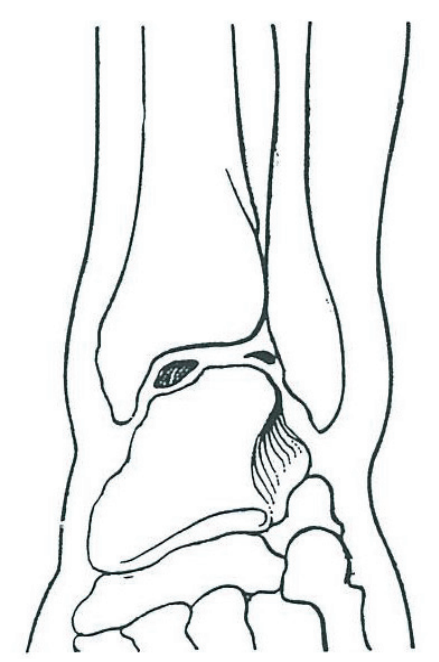

Figura 7. Morfología de las lesiones de la osteocondritis disecante de astrágalo. Las lesiones laterales son en forma de oblea y las lesiones mediales en forma de copa y mas profundas. realidad fracturas osteocondrales causadas por un traumatismo. Estos autores clasificaron las lesiones o fracturas en cuatro etapas diferentes (Figura 6): etapa I, pequeña área de compresión del hueso subcondral; etapa II, fragmento osteocondral parcialmente desprendido; etapa III, fragmento osteocondral completamente desprendido sin desplazamiento; etapa IV, fragmento osteocondral completamente desprendido con desplazamiento. En su estudio, el $43 \%$ de las lesiones eran localizadas en la parte lateral, por lo general en el tercio medio del astrágalo, mientras que el $57 \%$ se observaron en la porción media, por lo general en el tercio posterior. En los experimentos con cadáveres, la lesión lateral fue producida por inversión y fuerte flexión dorsal, y la lesión medial fue causada por inversión, flexión plantar, y rotación lateral de la tibia sobre el astrágalo. Los resultados del tratamiento, tanto en adultos y niños, fueron superiores en los pacientes tratados con cirugía en comparación con aquellos tratados de forma conservadora.

Más recientemente, Davidson et al. ${ }^{25}$ informaron de 27 fracturas transcondrales del astrágalo y se mostraron de acuerdo con Brendt y Harty en que el diagnóstico era el esperado con frecuencia y que una rápida intervención

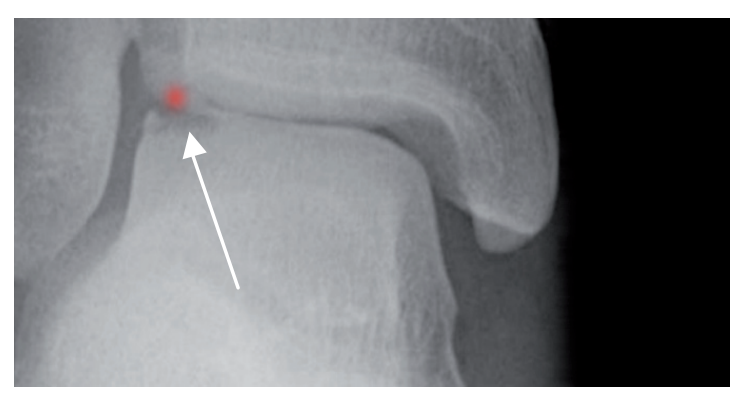


quirúrgica producía mejores resultados y prevenia la artrosis de tobillo.

Mukherjee y Young ${ }^{26}$ observaron resultados satisfactorios en 10 pequeñas lesiones sin desplazamiento después del tratamiento conservador. Ellos recomendaban extirpar los pequeños fragmentos desplazados y afirmaban que los grandes fragmentos desplazados se podían reparar con reducción abierta y fijación interna.

De las 31 lesiones tratadas en la clínica de Campbell ${ }^{27} 14$ eran mediales, 15 laterales y 2 centrales. Los síntomas comenzaron en la segunda década de vida en 21 pacientes. 25 de las lesiones -9 mediales, 15 laterales y 1 centralestaban asociadas con una historia de traumatismos. Una historia de lesión en pura inversión estaba relacionada con una de las lesiónes mediales y con 7 de las lesiones laterales. Dos de las lesiones laterales estaban asociadas con una lesión en eversión pura y flexión dorsal. Las 12 lesiones restantes se asociaron con un traumatismo aplastante o mal descritas como lesiones de torsión. De las lesiones sin historia de traumatismo, 5 fueron mediales y una central. Conforme a la clasificación de Brendt and Harty, no hubo lesiones en etapa I y sólo una lesión lateral fue de etapa II. De 24 lesiones en estadio III, 13

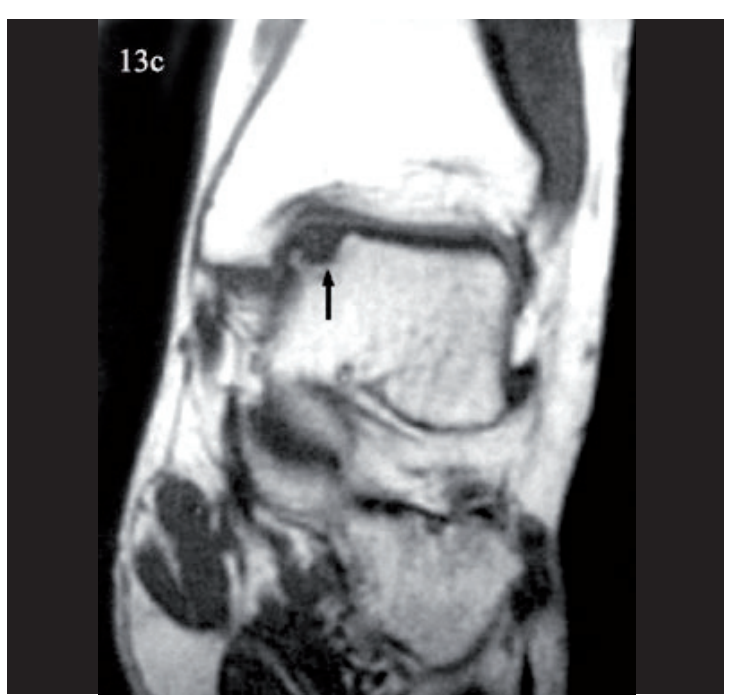

Figura 9. A. La RM pone de manfiesto el fragmento suelto en el cráter. eran mediales, 10 eran laterales y 1 era central. De las 6 lesiones en estado IV, una era medial, 4 eran laterales y una era central.

Morfológicamente, las lesiones mediales son más en forma de copa, más profundas, y se parecen más a una osteocondritis disecante. Las lesiones laterales están más en forma de oblea, son más superficiales, y se asemejan a una fractura osteocondral (Figura 11). Las lesiones mediales además, tienden a permanecer dentro del cráter y producir menos síntomas que las lesiones laterales que tienden a ser desplazadas.

\section{DIAGNÓSTICO}

Cuando un esguince de tobillo no se resuelve, debe tenerse en cuenta la posibilidad de que exista una fractura osteocondral de astrágalo. Baker et al. ${ }^{28}$ informaron que el $80 \%$ de los pacientes con osteocondritis disecante traumáticas tienen antecedentes de esguince de tobillo aparentemente benigno. Si hay derrame persistente, sinovitis retardada o bloqueo de la articulación, el examen radiográfico debe hacerse 4 o 5 semanas después de la lesión. La proyección

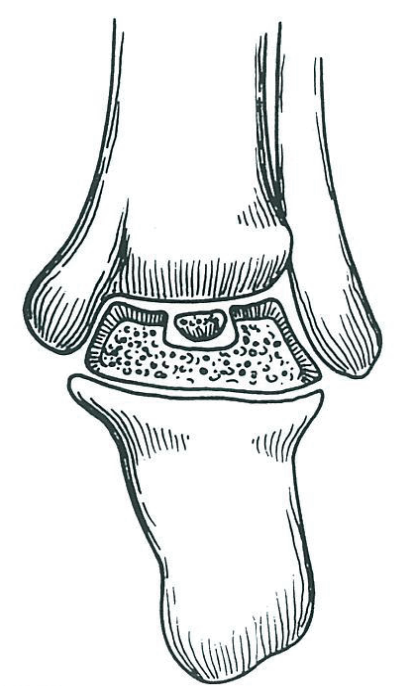

Figura 10. Posición del fragmento invertido dentro de la articulación del tobillo, el cartílago está apuesto al cráter y el hueso esponjoso hacia el cartílago articular tibial. 
oblicua y en flexión que eviten la superposición tibial y generalmente muestran la lesión con mayor claridad que las radiografías simples (Figura 8). Si se sospecha fractura osteocondral debe hacerse una gammagrafía ósea. Una vez que la lesión está localizada, las tomografías pueden determinar su profundidad y tamaño. La resonancia magnética es, en la actualidad, cara y probablemente no sea necesaria; sin embargo, se puede determinar si el fragmento esta unido o no al cráter (Figura 9). Si se requiere cirugía, la planificación preoperatoria puede ser ayudada por la tomografía computerizada; cortes axiales determinan la localización de la lesión (anterior, medial o posterior) y la necesidad de osteotomía del maléolo medial. Si el fragmento parece estar flotando en el cráter, por lo general se invierte de modo que el cartílago se coloca en dirección al cráter y hueso esponjoso se coloca hacia la articulación del tobillo (Figura 10). Las tomografías son útiles para la localización de la lesión flotante.

\section{TRATAMIENTO}

Las lesiones en estado I y algunas en estado II, sean mediales o laterales, suelen curarse espontáneamente o pueden ser tratadas de forma conservadora. Las lesiones en estado II y estado III mediales, que por lo general no están desplazadas en el cráter, tienen menos síntomas y causan con menos frecuencia artritis traumática que las lesiones en estado III laterales. Debido a esta clasificación, las lesiones mediales estables en etapa III,no desplazadas generalmente, se pueden tratar de forma conservadora, pero las lesiones laterales en etapa III a menudo requieren la escisión en los adultos, ya que tienen menos probabilidades de curarse, causar más síntomas, y a menudo dar lugar a artritis traumática ${ }^{29}$. En niños con fisis abiertas, sin embargo, un período de prueba de un tratamiento conservador es justificado para evitar la producción de un cráter en el astrágalo. El tratamiento no quirúrgico consiste en la aplicación de un yeso corto, una ortesis de pie tobillo, soportes para el arco, o botas de cuero con cordones por encima del tobillo. $\mathrm{Si}$ el dolor es intenso y la limitación para la actividad persiste después de al menos 6 meses de tratamiento conservador, está indicado el tratamiento quirúrgico.

Si el tratamiento quirúrgico es necesario, el fragmento puede extirparse por artrotomía o artroscopia. Parisien ${ }^{30,31}$, Andrews et al..$^{32}$, Drez et al. ${ }^{33}$, Guhl ${ }^{34}$, y Baker et al. ${ }^{28}$ describieron todas las técnicas artroscópicas para el tratamiento de osteocondritis disecante de la articulación del tobillo, pero dichas técnicas tienen cierta dificultad y requieren una experiencia considerable con la instrumentación artroscópica ${ }^{35}$. Una artrotomía bien realizada es preferible a un procedimiento artroscópico mal hecho. Si la extirpación de todo el fragmento no es segura después de la artroscopia, debe realizarse inmediatamente una artrotomía. Una pequeña artrotomía de tobillo de $5,08 \mathrm{~cm}$, tiene menos morbilidad que la artrotomía de la articulación de la rodilla y, a menudo se puede realizar como un procedimiento ambulatorio. Si se utilizan técnicas abiertas de artroscópia los mejores resultados se obtienen con la perforación y excisión del cráter.

Como la mayoría de las lesiones son pequeñas y la tibia se articula distalmente con los lados del cráter, la escisión del fragmento ha tenido éxito en aproximadamente el $87 \%$ de los pacientes con osteocondritis disecante de astrágalo. Ocasionalmente, si el fragmento es grande (mayor de $1.5 \mathrm{~cm}$ de diámetro) y es localizado en la superficie que soporta el peso de la articulación puede estar indicada la reducción abierta y fijación interna. El fragmento debe ser unido al hueso esponjoso subcondral viable en su superficie inferior para una mayor posibilidad de unión. Por el contrario, un cuerpo libre solamente cartílaginoso es menos probable que cure. Si el fragmento no cura, una segunda operación con fijación interna seria necesaria para eliminar el fragmento. Esto debe ser explicado al paciente antes de la cirugía. El enfoque preferido es a través de una artrotomía anterior $^{36}$. El fragmento se fija retrógradamente, no teniendo que utilizar técnicas de injerto, trabeculas óseas biodegradables, o la fijación mediante de tornillo Hebert.

La artrotomía se puede realizar sola o en conjunto con la artroscopia. Las lesiones laterales son más fácilmente expuestas que las lesiones mediales porque el maléolo lateral es más posterior que el maléolo medial. La 


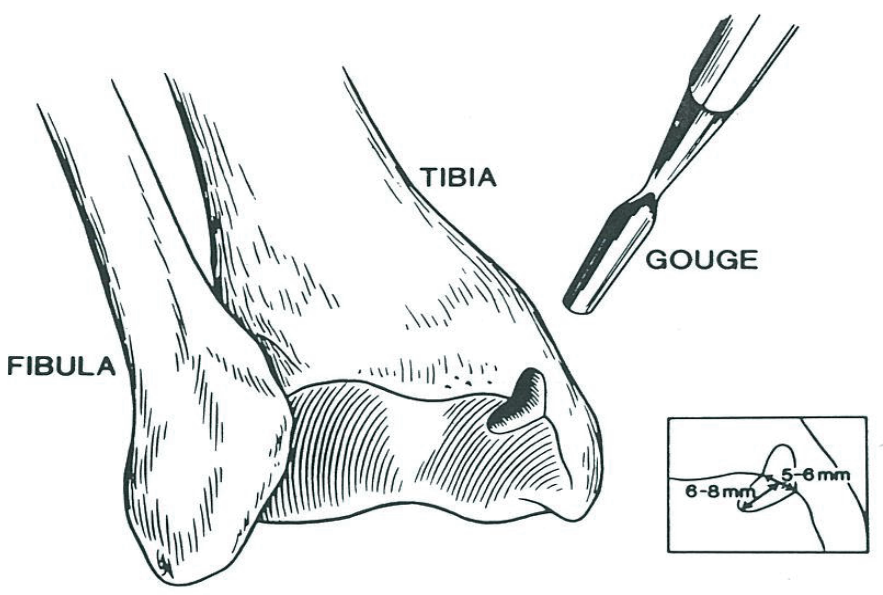

Figura 11. Técnica de ranurado (Flick and Gould) a nivel distal de la tibia para la exposición de las lesiones posteromediales sin osteotomía del maléolo medial. tomografía computerizada es útil en las lesiones localizadas a nivel medial; Si las lesiones son posteriores, puede ser necesaria una osteotomía del maléolo medial, pero esto debe evitarse si es posible cuando la fisis distal de la tibia está abierta. Colocar el pie en flexión plantar permite la inspección de las lesiones más posteriores en la cúpula del astrágalo. La osteotomía del maléolo medial se ha utilizado para este articulo, pero esta osteotomía del maléolo medial está plagada de complicaciones como la consolidación viciosa, pseudoartrosis, y la necesidad de extracción de los tornillos .La flexión plantar de los dedos de los pies se debe evitar para evitar daños en las estructuras neurovasculares dorsales y tendones, y la incisión debe extenderse cuidadosamente para evitar la rotura o corte de los tendones extensores. La artrotomía se realiza normalmente a través de un enfoque anteromedial, pero Thompson describe un enfoque posteromedial para ser usado si el fragmento osteocondral no puede ser expuesto a través de una incisión anteromedial. En esta técnica, un colgajo se repliega posteriormente a través de la incisión anteromedial, evitando dañar estructuras nerviosas, vasculares y tendinosas por detrás del maléolo medial, y se realiza una incision capsular para exponer la cara posterior del astrágalo. Flick y Gould ${ }^{37}$ describieron un "ranurado" tibial en la cual un área pequeña en la zona distal de la tibia es acanalada con una gubia para exponer la lesión posteromedial (Figura 11).
Aunque no parece haber ninguna otra articulación del cuerpo, donde se retire el cartílago para mejorar la exposición, es beneficioso en algunos pacientes aunque debe evitarse hasta que se conozca el efecto a largo plazo.

\section{TÉCNICA DE LA ARTROTOMÍA DE TOBILLO}

Si se hace después de la artroscopia, se aplica un segundo paño estéril, y se cambian guantes y bata continuando con la artrotomía a través del orificio anterolateral o anteromedial de la artroscopia. Si no se hace tras la artroscopia, se hace una incisión vertical de aproximadamente 1,5 centímetros de largo a nivel anterolateral o anteromedial, teniendo cuidado con los tendones extensores y el paquete neurovascular. El pie se colocará en una posición de flexión plantar para exponer la lesión, que se escindirá con una pinza o cureta. La zona se raspará hasta el hueso sangrante, y se perforarán tres o cuatro agujeros con una broca pequeña. Esto se puede lograr a través de un enfoque transmaleolar bajo visión directa si es necesario. La osteotomía del maléolo medial rara vez es necesaria y se requiere principalmente para lesiones mediales. Si es necesaria, el maléolo medial se perforará con un tornillo canulado de 4.5 o 6.0 (Figura $12 \mathrm{~A}$ ) continuando con la osteotomía oblicua del maléolo medial a través del hueso metafisario y retrayéndolo para exponer la lesión (Figura 12 B). Cuando la lesión 



Figura 12. Artrotomía del tobillo. A, perforación del maléolo medial. B, fragmento desplazado en la osteotomía para exponer la lesión. C, fijación interna con tobillo de hueso esponjoso.


Figura 13. A. Exposición anteromedial del astrágalo con el pie en máxima flexión plantar. B. Exposisión posteromedial a través de la incisión de la piel con el pie en posición de flexión dorsal máxima. 
haya sido resecada y el cráter perforado, el sitio de la osteotomía se fija con el tornillo de hueso esponjoso (Figura 12 C). Para finalizar se cerrarán las heridas colocando un apósito estéril e inmovilizando con un yeso hasta la altura del tendón rotuliano.

Retorno a la actividad deportiva. Después de 2 semanas de inmovilización con el yeso se retirará este y las suturas, y se colocará una férula de tobillo-pie durante 10 semanas. La restricción de las actividades deportivas puede ser necesaria si se realiza la osteotomía del maléolo medial. La vuelta a la actividad deportiva suele ser generalmente a las 4-6 semanas.

\section{TÉCNICA DE THOMPSON Y LOO- MER $^{38}$}

Se realiza una incisión curva, convexa y centrada de $10 \mathrm{~cm}$ tras el maléolo medial, exponiendo así la capsula medial. A continuación se realiza una incisión longitudinal de $2 \mathrm{~cm}$ en la cápsula a nivel anteromedial que se extenderá desde la tibia al astrágalo (Figura $13 \mathrm{~A}$ ). El pie estará posicionado en flexión plantar máxima observando así la parte superomedial de la cúpula astragalina. Si el defecto no puede ser inspeccionado completamente, se realizará un curetaje y perforado desde este enfoque realizando una incisión curva sobre el tendón tibial posterior. Se retraerá anteriormente, y la incisión se hará en la superficie profunda del retináculo flexor (Figura $13 \mathrm{~B}$ ). La retracción posterior se hará suavemente, retrayendo los contenidos del tú- nel tarsiano sin que sean expuestos. Teniendo en cuenta lo anterior, el pie tomará posición de flexión dorsal, pudiendo observarse la parte superomedial de la cúpula astragalina; tras esto, la lesión será observada adecuadamente y tratada por escisión o curetaje.

Retorno a la actividad deportiva. Tras la cirugía se iniciarán ejercicios de movimiento articular, y a deambular tan pronto como sea tolerado por parte del paciente. El regreso a la actividad deportiva suele ser entre las 12-16 semanas.

\section{CONCLUSIONES}

- Existen numerosas lesiones en el astrágalo que pueden confundirse con esguinces de tobillo, y es importante tener esto en cuenta para no tratarlas como si de un esguince se tratara; para ello, deben hacerse pruebas diagnósticas como la resonancia magnética, la radiografía o la gammagrafía ósea, de tal forma que sea más fácil dar un diagnóstico correcto.

- Hay que tener en cuenta,sobre todo a los pacientes jóvenes y deportistas ya que es en este colectivo donde se producen con más frecuencia estas lesiones.

- Es de gran importancia establecer un diagnóstico precoz en las lesiones de astrágalo, con el fin de poder instaurar un tratamiento conservador y evitar el desarrollo de procesos degenerativos o artrósicos que hagan necesaria la aplicación de tratamientos quirúrgicos o invasivos.

\section{BIBLIOGRAFÍA}

1. Amis, J. A., and Gangl, P. M. When inversion injury is more than a "sprained ankle."J Musculoskel Med. 1987, pp.68-70.

2. McManama, G. B. Ankle injuries in the young athlete. Clin Sports Med .1988, 7(3):547-62.

3. Snyder, R. B., Lipscomb A. B., and Johnston, R. K. The relationship of tarsal coalitions to ankle sprains in athletes. Am J Sports Med. 1981; 9(5):313-7.

4. O'Neil, D. B., Micheli L.J. Tarsal coalition: A followup of adolescent athletes. Am J Sports Med. 1989;17(4):544-9.

5. O'Donoghue, D. H.Inpingement exostosis of the talus and tibia. J Bone Joint Surg. 1957;39(A):835.

6. Hontas, M. J., Haddad, R. J., Schlesinger, L. C. Conditions of the talus in the runner. Am J Sports Med.1986;14(6):486-90.

7. Kleiger, B. Anterior tibiotalar impingement syndromes in dancers. Foot Ankle.1982;3(2):69-73.

8. Parkes, J.C., Hamilton, W. G., Patterson, A.H., and Rawles, J. G. The anterior impingement syndrome of the ankle. J Trauma. 1980;20(10):895-8.

9. Brodsky, A. E., Khalil, M. A. Talar compression syndrome. Am J Sports Med.1986;14(6):472-6. 
10. Waller, J. F., Jr. Hindfoot and midfoot problems of the runner. Symposium on the Foot and Leg in Running Sports.1982. St. Louis, C. V. Mosby.

11. Ecker, M. L., Ritter, M. A., and Jacobs, B. S. The Symptomatic Os Trigonum. JAMA.1967;201(11):882.

12. Johnson, R. P., Collier, B. D., and Carrera, G. F. The os trigonum syndrome: use of bone scan in the diagnosis. J Trauma. 1984;24(8):761-4.

13. Mc Dougall, A. The os trigonum. J bone Joint Surg. 1965;37(B):257-65.

14. Stieda, J. H. Talus und das os trigonum bardelebens beim Menschen. Anat anz. 1889. 4:305-315.

15. Rosenmuller, P., quoted in Holland, C. T. On rarer ossifications seen during X-ray examinations. J Anat. 1921;55:235-40.

16. Shepherd, F. J. A Hitherto Undescribed Fracture of the Astragalus. J Anat Physiol. 1882. 17:79-81.

17. Turner, W. A. A Secondary Astragalus in the Human Foot. J Anat Physiol. 1882;17:82-3.

18. Watson, C. A., and Dobas, D. C. The os trigonum: a discussion and case report. Arch Podiatr Med Foot Surg. 1976;3:17-21.

19. Grogan, D. P., Walling, A. K., and Ogden, J. A. Anatomy of the os trigonum. J Pediatr Orthop.1989;10(5):618-22.

20. Konig, F. Ueber freie Korper in den Gelenken. Dche Zschr Chir. 1888 ;27(1-2):90-109.

21. Kappis, M. Weitere Beiträge zur traumatisch-mechanischen Entstehung der „spontanen” Knorpelablösungen (sogen. Osteochondritis dissecans). Dtsch Zschr Chir. 1922;171(1-2):13-29.

22. Rendu, A. Fracture intra-articulaire parcellaire de la poulie astraglienne. Lyon Med. 1932;150-220.

23. Roden, S., Tillegard, P., and Unander-scharin, L. Osteochondritis dissecans and similar lesions of the talus: report of fifty-five cases with special reference to etiology and treatment. Acta Orthop Scand. 1953;23(1):51-66.

24. Berndt, A. L. and Harty, M. Transchondral fracture of the talus. J Bone Joint Surg. 1959;41(A):9881029.

25. Davidson, A. M., Steele, H. D., MacKenzie, D. A., and Penny, J. A. A review of twenty-one cases of transchondral fracture of the talus. J Trauma. 1967;7(3):378-415.

26. Mukherjee, S. K., and Young A. B. Dome fractures of the talus :report of ten cases. J Bone Joint Surg. 1973;55(b):319.

27. Canale, S. T., and Beaty, J. Osteochondral lesions of the talus.In Hamilton, W. C. (Ed),Traumatic Disorders of the Ankle. New York, Springer-Verlag, 1984. 1984;

28. Baker, C. L., Andrews, J.R., and Ryan, J. B. Arthroscopic treatment of transchondral talar dome fractures. Arthroscopy. 1986; 2:82.

29. Lehman, R. C., and Gregg, J.R. Osteochondritis dissecans of the midfoot. Foot Ankle. 1986;7(3):17782.

30. Parisien, J. S. Arthroscopic treatment of osteochondral lesions of the talus. Am J Sports Med.1986];14(3):211-7.

31. Parisien, J. S. Diagnostic and operative arthroscopy of the ankle technique and indications. Bull Hosp Joint Dis.1985;45(1):38-47.

32. Andrews, J. R., Previte, W. J., and Carson, W. G. Arthroscopy of the ankle: technique and normal anatomy. Foot Ankle. 1985;6(1):29-33.

33. Drez, D., Guhl, J. F., and Gollehon, D. L. Ankle Arthroscopy: Technique and Indications. Foot Ankle. 1981;2(3):138-43.

34. Guhl, J. F. New techniques for arthroscopic surgery of the ankle: preliminary report. Orthopedics. 1986;9(2):261-9.

35. Martin, D. F., Baker, C. L., Curl, W. W., Andrews, J. R., Robie, D. B., and Haas, A. F. Operative ankle arthroscopy. Long-term followup. Am J Sports Med.1989; 17(1):16-23;

36. Yocum, A. L. Treatment of osteochondritis dissecans of the talus. In Shields, C. L., Jr. (Ed.), Manual of Sports Surgery. New York, Springer-Verlag, 1987.

37. Flick, A. B.,and Gould, N. Osteochondritis dissecans of the talus (transchondral fractures of the talus): review of the literature and new surgical approach for medial dome lesions. Foot Ankle.1985;5(4):165-85.

38. Thompson, J. P., and Loomer, R. L. Osteochondral lesions of the talus in a sports medicine clinic. A new radiographic technique and surgical approach. Am J Sports Med. 1984;12(6):460-3. 